
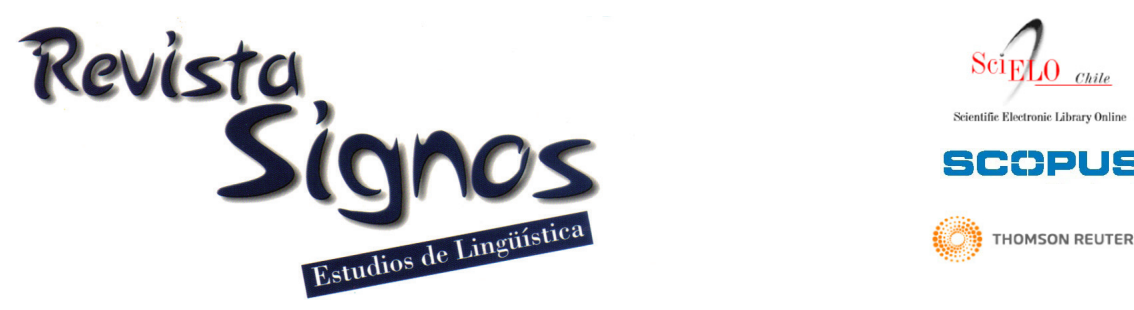

\title{
La voz del autor en la escritura académica: Una propuesta para su análisis'
}

\section{Authorial voice in academic writing:A methodological proposal for its analysis}

Montserrat Castelló

montserratcb@blanquerna.url.edu Universitat Ramon Llull España

\author{
Mariona Corcelles \\ marionacs@blanquerna.url.es \\ Universitat Ramon Llull \\ España
}

\author{
Anna Iñesta \\ ana.inesta@esade.edu \\ Universitat Ramon Llull \\ España
}

\author{
Gerardo Bañales \\ gvanales@uat.edu.mx \\ Universidad Autónoma de Tamaulipas \\ México
}

\author{
Norma Vega \\ navegalo@uat.edu.mx \\ Universidad Autónoma de Tamaulipas \\ México
}

Recibido: 26-III-20I0 / Aceptado: |8-I-20I |

\begin{abstract}
Resumen: La escritura académica ha perdido gradualmente su etiqueta tradicional de discurso objetivo e impersonal y se ha convertido en una empresa persuasiva que implica la interacción entre escritor y lector. Sin embargo, la noción de voz dista mucho aún de ser un concepto unívoco y su significado varía en función de la perspectiva desde la que se aborde su estudio. Los problemas atañen tanto a su definición, a las relaciones de la noción de voz con otros conceptos afines como a problemas metodológicos respecto a las dimensiones necesarias y suficientes para abordar su estudio y los indicadores relevantes a la hora de observar, analizar y valorar su presencia en un texto determinado. El propósito de este artículo es triple. En primer lugar, pretendemos revisar los trabajos que en los últimos años se han ocupado del estudio de la voz en los textos, delimitando desde qué orientaciones disciplinares y teóricas han abordado este estudio; nuestra revisión no pretende ser exhaustiva sino representativa de los trabajos más relevantes de cada perspectiva teórica. En segundo lugar, presentamos nuestra aproximación conceptual a la noción de voz en base al análisis y la discusión sobre las aportaciones anteriores. En último término, se ofrece una propuesta metodológica que contempla tres dimensiones de análisis estrechamente relacionadas pero independientes, cada una de ellas centrada en los aspectos que, de acuerdo a la revisión efectuada, consideramos relevantes en el estudio de la noción de voz.
\end{abstract}

Palabras Clave: Voz académica, autoría, escritura académica, posicionamiento, intertextualidad. 


\begin{abstract}
Academic writing has gradually lost its traditional label of objective and impersonal discourse becoming a persuasive endeavour involving the interaction between writer and reader. Despite its popularity, the notion of voice is far from being a univocal concept and its meaning varies according to the perspective adopted. Problems involve its definition, the relationships with other related concepts, and methodological issues concerning the appropriate dimensions and the relevant aspects that should be addressed when observing, analysing and assessing traces of voice in a specific text. The paper has three main objects. Firstly, we revise research studies that in the last ten years have devoted their efforts to clarify the notion of voice considering their conceptual, theoretical and disciplinary framework. Our revision does not intend to be exhaustive but representative of the studies carried out from each theoretical perspective and its main findings. Our second aim is to build our own perspective of voice integrating the findings of previous research. Finally, a methodological proposal is established detailing three dimensions of analysis which are closely related, each one of them focused on the aspects that, according to the revision, we consider relevant for the study of voice.
\end{abstract}

Key Words: Academic voice, authorial identity, academic writing, stance, intertextuality.

\section{INTRODUCCIÓN}

En los contextos educativos de secundaria y universidad, es habitual que los profesores exijan a sus estudiantes escribir textos complejos que requieren algo más que resumir o sintetizar de forma impersonal la información (Castelló, Iñesta, Miras, Solé, Teberosky \& Zannoto, 2007). La escritura académica ha perdido gradualmente su etiqueta tradicional de discurso objetivo e impersonal y se ha convertido en una empresa persuasiva que implica la interacción entre escritor y lector (Hyland, 2005). En este sentido, cada vez se valora más que los estudiantes adopten una posición con respecto a los temas sobre los que escriben, es decir, que construyan su propia voz y la reflejen o la trasladen al texto. Sin embargo, la noción de voz, a pesar de su popularidad, dista mucho de ser un concepto unívoco y su significado varía en función de la perspectiva desde la que se aborde su estudio. Los problemas atañen tanto a su definición $y$, consiguientemente, a la explicación acerca de cómo los escritores son capaces de construir la propia voz en un texto, como a las relaciones de la noción de voz con otros conceptos afines tales como los que se refieren a la identidad del escritor o al concepto de autoría.

Estas dificultades en cuanto a la delimitación teórica se traducen, además, en problemas metodológicos respecto a las dimensiones necesarias y suficientes para abordar su estudio, así como a la consideración de cuáles son los indicadores (discursivos, sociales - psicológicos) relevantes a la hora de observar, analizar y valorar su presencia en un texto determinado.

El propósito de este artículo es triple. En primer lugar, pretendemos revisar los trabajos que en los últimos años se han ocupado del estudio de la voz en los textos, delimitando desde qué orientaciones disciplinares y teóricas han abordado este estudio; nuestra revisión no pretende ser exhaustiva sino representativa de los trabajos más relevantes de cada perspectiva teórica. En segundo lugar, aspiramos a clarificar nuestra aproximación conceptual a la noción de voz en base al análisis y la discusión sobre las aportaciones anteriores. En último término, presentamos una propuesta metodológica que contempla tres dimensiones de análisis estrechamente relacionadas pero independientes, cada una de ellas centrada en los aspectos que, de acuerdo a la revisión efectuada, consideramos relevantes en el estudio de la noción de voz.

\section{Diferentes enfoques en el estudio de la voz en los textos académicos: Aportaciones desde la Lingüística, la Psicología y la literacidad crítica}

Los autores que han abordado de forma más decidida el estudio de la voz en los textos académicos pueden circunscribirse a tres grandes perspectivas que, a su vez, se corresponden con determinados enfoques teóricos y opciones metodológicas.

En primer lugar, destacan las aportaciones de la lingüística, en especial de la pragmática y el análisis textual. No podemos -ni es nuestro propósitorevisar aquí el ingente número de trabajos generados desde esta perspectiva, por lo que nos limitaremos a exponer las características compartidas por la mayoría de estos trabajos, analizando más detenidamente las aportaciones de aquellos autores más reconocidos y cercanos a nuestros intereses. 
Desde esta perspectiva, se considera que el antecedente y el origen de la reflexión acerca de la presencia del autor en sus textos es la teoría de la enunciación. Benveniste (1970) la define como:

“proceso de 'apropiación'. El locutor se apropia del aparato formal de la lengua y enuncia su posición de locutor por medio de indicios específicos, de un lado, y de procedimientos accesorios, de otro. Pero inmediatamente, desde el preciso momento en que se declara locutor y asume la lengua, implanta al 'otro' ante sí mismo, sea cual sea el grado de presencia que atribuya a este otro" (énfasis original) (Calsamiglia \& Tusón, 2007: 124).

Los procedimientos específicos y accesorios para señalar la posición del autor -a los que se refiere Benveniste (1970)- son el principal foco de interés de los estudios desarrollados por Hyland (2002). La investigación de este autor, a quien podemos considerar uno de los representantes más destacados de esta perspectiva, se centra en los mecanismos discursivos que el escritor utiliza para posicionarse en sus textos. Dicho posicionamiento es indispensable en el caso de los textos académicos ya que el análisis y la interpretación de la información que les son característicos requieren la construcción de un punto de vista personal. Así, a la hora de escribir este tipo de textos, los estudiantes deben situarse en relación al material que están discutiendo y encontrar una manera satisfactoria de expresar sus propias afirmaciones y argumentos (Cadman, 1997). Hyland (2002) considera que para marcar su posición, los escritores de textos académicos deben ir más allá de sus proposiciones, conseguir que esa posición sea visible a través de las palabras. En sus estudios además, asume que los mecanismos para posicionarse son dependientes del contexto, situados, y por tanto, específicos en función de las disciplinas en las que se escribe y en función de los objetivos comunicativos que cada texto persiga.

Precisamente, los trabajos desarrollados por Hyland a lo largo de los últimos diez años han proporcionado datos muy valiosos para avanzar en el conocimiento de las especificidades de los textos académicos y sus discursos en diferentes disciplinas. Su propuesta metodológica -que analizaremos con más detalle en el siguiente apartado- se centra inicial y preferentemente en el análisis de las herramientas discursivas que permiten identificar la posición del autor, que le confieren autoridad o le permiten ser aceptado y convencer a sus lectores.
Sin embargo, consistente con la idea de que los textos se insieren en contextos sociales y discursivos que implícitamente limitan el abanico de posibilidades expresivas para los autores, en sus investigaciones recientes incluye también el análisis de la perspectiva de los autores con el objetivo de indagar

"su grado de entendimiento sobre la efectividad retórica de los textos y sobre sus propias preferencias y prácticas discursivas" (Hyland, 2005: 178).

Los resultados de estos trabajos ponen de manifiesto que los escritores se representan a sí mismos de formas diferentes según la disciplina a la que pertenecen (Hyland, 2005). Así, en ciencias sociales los escritores de textos académicos creen necesaria y pertinente la explicitación de sus posiciones personales, mientras que en las ciencias llamadas 'duras' como las ingenierías tienden a primar los mecanismos orientados a la demostración y a la generalización evitando las interpretaciones personales. En este sentido, los datos apoyan la consideración de la escritura como un proceso de enculturación en el uso de los géneros y de las herramientas discursivas propias de cada disciplina, a la vez que constituye un instrumento para la construcción de la propia identidad como miembros de dicha disciplina. Estos resultados, unidos a los análisis de las características específicas de un corpus de más de doscientos textos escritos en ocho disciplinas diferentes, le permiten afirmar que existen estrechas relaciones entre el concepto de voz en los textos y la construcción de una identidad profesional (Hyland, 1999, 200 I).

Entre sus conclusiones, resulta significativa la imposibilidad de 'borrar' la presencia del autor ni tan siquiera en aquellas disciplinas en las que se supone que no es conveniente explicitar las interpretaciones personales. Los textos analizados revelan que las herramientas discursivas empleadas para conseguir que esta voz sea visible en el texto permiten modalizar, expresar puntos de vista con precisión, cautela y diplomacia de forma diferente pero imprescindible en cada una de las disciplinas. Así, por ejemplo, el autor demuestra cómo los escritores usan estos mecanismos en textos académicos de biología molecular para marcar su posición ante el lector o cómo recurren de forma habitual al uso de la primera persona o introducen autorreferencias en la mayoría de las disciplinas analizadas. 
En sus últimas aportaciones, es evidente el avance hacia la consideración de la voz como una característica que trasciende los recursos lingüísticos y que compromete a toda la persona. En sus propias palabras:

"la creación de la persona del autor (authorial persona) es un acto de elección personal, y la influencia de la personalidad individual, la confianza, la experiencia, la preferencia ideológica son importantes" (Hyland, 2005: 191).

El segundo de los enfoques a los que hacíamos referencia al inicio de este apartado es el que desde la psicolingüística y la literacidad crítica se ha interesado por los fenómenos de la voz y la intertextualidad y sus relaciones con la construcción social de la identidad letrada en diferentes comunidades discursivas.

Uno de los autores más relevantes de esta corriente, Gee (2000), defiende que los usos del lenguaje construyen una determinada imagen de nosotros que deviene reconocible a los ojos de los demás. Hablamos y escribimos de forma diferente según las situaciones y los interlocutores reales o imaginarios, lo que equivale a afirmar que las prácticas discursivas son siempre situadas y que la forma en la que participamos en los discursos tiene consecuencias en nuestra actividad académica, profesional y personal, en definitiva en nuestra identidad. Según este autor, las personas disponemos de un 'kit identitario', es decir, de un conjunto de formas y combinaciones de hablar, escribir, movernos, etc., que nos identifican y nos marcan como miembros de una comunidad en un determinado momento (Gee, 2005, 2006). Afirma también que esta identidad puede ser diferente en función de las situaciones y de las comunidades a las que un mismo sujeto pertenece.

Desde una posición teórica cercana, pero más vinculada a los postulados de la literacidad crítica, Ivanic $(1998,2005)$ también establece esta relación entre voz e identidad, si bien la autora se refiere preferentemente a los procesos de construcción de la identidad social. Según su punto de vista, la escritura siempre vehiculiza una determinada voz que sitúa al escritor en unos determinados discursos sociales y culturales. La escritura obliga a realizar determinadas elecciones discursivas (según valores, intereses, creencias, prácticas y relaciones de poder) y a desestimar otras. Desde este punto de vista, escribir en contextos académicos implica recurrir y dialogar con los discursos ya existentes de manera intencional de forma que dicha combinación personalizada de los discursos sociales pondría de manifiesto la identidad del autor. De manera parecida a Gee (2005), Ivanic $(1998,2005)$ considera la escritura como una actividad situada y entiende que la voz del escritor es el resultado de la orquestación de las diferentes voces presentes en los discursos sociales en función de la situación comunicativa en la que se inserta.

De acuerdo con esta autora, la identidad de un escritor se desplegaría en tres dimensiones interrelacionadas que se activarían de forma complementaria en el momento de escribir:

- el yo autobiográfico, que se refiere a lo que el escritor aporta al acto de escribir, los recursos de que dispone y su historia previa como escritor;

- el yo discursivo que se construye mientras se está escribiendo. Implica la identificación con determinados discursos sociales que en muchas ocasiones se produce de forma implícita;

- el yo autor que también se construye durante el acto de escribir pero que implica un mayor nivel de explicitud y que conlleva el sentimiento de autoría, la percepción de que el autor dialoga con otras voces para crear un discurso propio.

A estas dimensiones habría que sumarle la dimensión social que influye en la manera como el escritor se representa a los potenciales lectores y su influencia en el manejo de la propia voz (más o menos dialógica, explícita, etc.).

El concepto de voz en el texto así entendido tendría una dimensión individual y una dimensión social (implica toma de postura -monólogica- y discusión con otras posturas y discursos-dialógica).

En el ámbito de la psicología, y de lo que en EEUU se considera los estudios sobre composición escrita, el estudio de la voz en los textos ha estado preferentemente vinculado a la perspectiva sociocultural y a los postulados que relacionan la voz con el desarrollo de la conciencia (Farmer, 1995). Desde esta perspectiva, la construcción de una voz propia es posible gracias a la interiorización de otras voces que nos han acompañado en el proceso. Además, siguiendo a Bakhtin (1986), una voz nunca existe de forma aislada y cualquier enunciado es siempre multivocal. En base a estas 
premisas, Farmer (1995) cuestiona el concepto de voz propia entendida como verdadera (true voice). Para este autor, cada escritor tiene a su disposición un coro de voces, todas ellas 'verdaderas' (true voices) porque todas ellas son propias y de lo que se trata es de utilizar una u otra de estas voces en función de las características de cada situación discursiva. Desde el punto de vista educativo, sería pertinente ayudar a los estudiantes a apropiarse de las voces de otros para dotarlas de sentido personal y poder utilizarlas de forma intencional en sus textos.

La apropiación está, por tanto, directamente vinculada con la construcción de la propia voz. Según Farmer (1995), Bakthin (1986) utiliza el concepto de 'asimilación' para explicar el proceso de interiorización de Vygotsky, pero a diferencia de este introduce no solo el énfasis en la visión dialógica sino en la idea de esfuerzo (struggle). Se trata del esfuerzo que se requiere para dominar los significados de los demás, para hacerlos propios, para pasar, en definitiva de la propiedad colectiva a la propiedad privada en el uso del lenguaje. Esta no es una tarea fácil porque el lenguaje está superpoblado de intenciones y se requiere un esfuerzo crítico de 'expropiación' para que los significados de otros pasen a ser parte de las intenciones personales y puedan ser usados intencionalmente en contextos diversos generando nuevos significados (Corcelles, 2010).

Es precisamente en este esfuerzo de expropiación en el que aparece el concepto de autoría que desde esta perspectiva puede relacionarse con el de voz, en tanto esta es siempre una característica de los textos escritos por autores que se diferencian de otras voces pero que también dialogan con ellas. También Prior $(1998,200 I)$ define la voz como una forma de reinvocar las voces de otros, no solo a través de la intertextualidad, sino como una forma de asegurar la identidad social del escritor que se alínea con unos discursos particulares y participa en determinadas comunidades discursivas (Lensmire, 1998). De forma parecida se expresa Bazerman (2004) cuando afirma que la identidad se construye discursivamente y consecuentemente la voz en los textos no debería estudiarse solo a través del análisis de los textos a los que el autor hace referencia, sino a la luz de cómo los interpreta y los utiliza haciendo un énfasis especial en la intencionalidad que le lleva a adoptar una determinada posición.

También desde una perspectiva psicológica sociocultural, Spivey (1997) defiende la representación del discurso académico como una amplia red de textos interrelacionados. Dentro de esta red 'intertextual', los escritores construyen 'su identidad de autor' (author's identity) o 'voz', mediante las conexiones dialógicas que establecen con los textos de otros autores. Además, esta autora también concibe el proceso de construcción de la identidad o voz de un autor no solo como un proceso individual que depende de las conexiones que el escritor pueda establecer, sino que los lectores participan en este proceso mediante el uso e interpretación de los textos del autor, citándolos, evaluándolos, criticándolos. De esta manera, un escritor se identifica a sí mismo y al mismo tiempo los demás también pueden identificar su voz entre aquellas que pueblan un 'territorio textual', en el que conviven unas determinadas comunidades discursivas situadas en periodos socioculturales e históricos específicos (Nelson, 200I; Nelson \& Castelló, en prensa). Desde esta perspectiva pues, la intertextualidad se considera tanto un proceso individual como social y se constituye como una dimensión imprescindible no solo para comprender sino para estudiar empíricamente la identidad o voz del autor.

Las conceptualizaciones de la voz que se proponen desde las perspectivas revisadas perfilan la escritura como una actividad extremadamente compleja que, siguiendo a Reuter y Lahanier-Reuter (2007) consideramos configurada por la modalidad (que conlleva una serie de restricciones y se traduce en la producción de un texto), la articulación de las diferentes dimensiones implicadas en un acto de producción (lingüística, cognitiva, afectiva, sociocultural...), la actualización en forma de texto, la integración de una esfera de prácticas socioinstitucionales, el hecho de que los escritores se sitúen en un contexto histórico y cultural individual y colectivo, las relaciones que se establecen entre esta y otras prácticas, y el significado que el escritor le atribuye. Se trata, además, de una práctica que modula o da forma a las actividades y estructura su significado, la representación de los participantes, así como las relaciones que se establecen entre la escritura y el conocimiento.

En este contexto, desde nuestra perspectiva la voz deviene en un constructo de carácter individual así como social, cultural e históricamente situado. Concretamente, la dimensión individual está relacionada con el hecho de que la presencia del autor se pone de manifiesto a través de determinados usos estratégicos del lenguaje, vinculados al proceso de 
enunciación (Beneviste, 1970, citado en Calsamiglia \& Tusón, 2007) ○ posicionamiento (Hyland, 2002). Complementariamente, la dimensión social y situada tiene que ver con la conciencia del autor sobre el poder de sus palabras para representarle como miembro de una comunidad discursiva (Gee, $2000,2005,2006$ ) y, consecuentemente, sobre la necesidad de ajustar los propios discursos en función de los lectores objetivos y, por tanto, de las características del contexto en el que el diálogo en diferido deba tener lugar (Ivanic, 1998, 2005). La voz no solamente invoca las voces de aquellos otros discursos dominantes en el ámbito académico correspondiente (Prior, 1998, 200I) sino que la construcción de una voz propia requiere de la expropiación crítica de los discursos de otros para que puedan ser utilizados intencionalmente en la construcción de nuevos significados (Farmer, 1995; Corcelles, 2010) que deben inserirse y que, a su vez, reciben su sentido en el seno de la red de textos que pueblan el diálogo disciplinario.

\section{Aproximaciones metodológicas al estudio de la voz en los textos académicos}

Si bien es cierto que desde el punto de vista metodológico las aproximaciones teóricas analizadas han priorizado diferentes aspectos en el estudio de la voz, no lo es menos que, en los últimos años, los autores a los que nos hemos referido coinciden en la necesidad de abordar los aspectos lingüísticos, retóricos, sociales y psicológicos de forma integrada para comprender cómo se construye la voz en los textos académicos y fundamentalmente para saber cómo podemos enseñar a nuestros estudiantes a gestionarla.

Sin embargo, persisten algunas diferencias sustanciales. Así, los estudios realizados desde perspectivas lingüísticas se interesan de manera preferente por los recursos lingüísticos y retóricos que, según los autores, reciben diferentes denominaciones: appraisal (Martin, 2000), evaluation (Hunston \& Thompson, 2000) ○ posición (stance) (Hyland, 1999). La posición o el posicionamiento ha sido el más ampliamente utilizado, si bien no siempre con idéntico significado. Para algunos autores, la posición indica cómo las diferentes identidades son generadas por los discursos socialmente disponibles (Davies \& Harré, 1990; Fairclough, 1995), mientras que otros ciñen el estudio de la posición a las diferentes modalidades lingüísticas (Calsamiglia \& Tusón, 2007).
En los últimos años, Hyland (2005), consciente de la variabilidad de términos para referirse a los mismos fenómenos, se interesa por el estudio del metadiscurso. Su propuesta considera parte del metadiscurso las expresiones lingüísticas orientadas a señalar el posicionamiento del autor. Define el metadiscurso como:

"[...] the cover term for the self-reflective expressions used to negotiate interactional meanings in a text, assisting the writer (or speaker) to express a viewpoint and engage with readers as members of a particular community" (Hyland, 2005: 37).

Para operativizar su estudio, establece dos dimensiones, interactiva e interaccional, que resumimos a continuación.

a) Dimensión interactiva: El objetivo principal del autor es el de poner límites a la interpretación del texto de manera que se ajuste a las necesidades de los lectores. Esto implica utilizar argumentos y recursos que permitan a los lectores recuperar el significado preferido por el autor.

b) Dimensión interaccional: El objetivo del autor en este caso se cifra en explicitar sus puntos de vista e involucrar a los lectores, permitiéndoles responder al texto que se va construyendo. Es la expresión de la 'voz' del escritor en tanto que personalidad reconocida en la comunidad. En este caso el metadiscurso es esencialmente evaluativo y de complicidad, expresando solidaridad o acuerdo, anticipando objeciones, respondiendo, en definitiva, a un diálogo imaginado con los lectores.

Desde su perspectiva, también Ivanic (2005) ha abogado en los últimos años por ampliar el tipo de datos relevantes en el estudio de la voz y pasar de metodologías exclusivamente centradas en los productos a análisis que también incluyan la perspectiva del escritor. Así, el uso de entrevistas le permite explorar los orígenes y las razones que explican la elección de determinadas palabras y frases. La autora se refiere al 'bricolaje discursivo' como un proceso a estudiar, porque revela cómo el escritor fue capaz de construir estructuras textuales complejas, pero también complejas identidades negociadas (Ivanic, 1998). Las entrevistas pues, le sirven, en último término para estudiar las relaciones entre consciencia y control de la identidad proyectada en los textos (Ivanic \& Camps, 200I). 
Desde una aproximación metodológica similar Kamberelis y Scott (1992), interesados por el estudio de la intertextualidad y la presencia de diferentes voces en un mismo texto, defienden la necesidad de analizar los textos vinculándolos con los contextos de producción. Así, para estudiar los textos de estudiantes de primaria, proponen entrevistar a los padres, profesores, además de realizar observaciones en el aula. Todos estos datos les permiten rastrear cómo se manifiestan las diferentes voces en los textos. Su análisis además vincula las relaciones intertextuales con diferentes ideologías políticas dado que la postura crítica en el análisis del discurso en la que se sitúan, cercana a la semiótica social, parte de la premisa de que es necesario relacionar el texto no solo con los contextos inmediatos de producción, sino también con un contexto social más amplio que hace referencia a las cosmovisiones o discursos sociales en conflicto que pueden manifestarse en el propio texto (Ivanic, 2005). Para estos autores, escribir y reescribir es como 'escribirse' y 'reescribirse' y, por lo tanto, el texto revela la identidad del escritor. En el ámbito de secundaria y desde la perspectiva sociocultural, Donahue (2005) estudia los cambios en los textos que los estudiantes escriben a partir de lecturas que actúan como textos-fuente. Su análisis se basa en las acciones denominadas de reprise-modification que dan cuenta de las ideas de los textos-fuente que se incluyen en el propio texto a la vez que describen el tipo y la cantidad de modificaciones que se realizan sobre la información original. En último término se supone que las modificaciones en las ideas de los textos-fuente y la aparición de nuevas ideas en los escritos de los estudiantes reflejan aspectos relevantes de la construcción de su identidad como escritores.

Desde la misma orientación teórica, Prior (1998) incluye de forma preeminente al escritor en su aproximación metodológica y propone el estudio de casos múltiples para indagar en las trayectorias de los estudiantes-escritores, analizar su implicación en diversas comunidades de práctica y relacionar estas trayectorias con sus procesos de composición. Concretamente, en sus trabajos con estudiantes de doctorado concluye que la escritura más literal - personalizada se relaciona con la menor o mayor implicación personal del estudiante en una comunidad de práctica.

\section{Una propuesta conceptual y metodológica integradora para el estudio de la voz en los textos académicos}

La consideración de la escritura académica como una actividad cognitiva y social implica que los procesos de composición se desarrollan de forma situada y dependiente de los contextos discursivos en los que los textos se inscriben. La forma en que los sujetos participan en los discursos y en los géneros discursivos tiene consecuencias en la construcción de su identidad social y, más específicamente, de su identidad como escritor (Gee, 2005; Ivanic, 2005). Esta última se pone de manifiesto a través de las elecciones discursivas del autor, cuya gestión estratégica implica la posibilidad de que el autor se sitúe de forma diferente en contextos diversos y utilice su(s) voz(ces) de forma diversa e intencional ante situaciones y demandas específicas.

En línea con los autores revisados, desde el punto de vista conceptual entendemos que la 'voz' de un escritor es a la vez individual y social $y$, dado que su manifestación varía, puede considerarse siempre situada cultural e históricamente. En términos de proceso, las características de la voz se traducen en el uso intencional de determinados recursos discursivos, en el diálogo y las relaciones intertextuales que el escritor establece con otros textos, y en las formas de organización de la información que otorgan una u otra estructura a los textos. Esta caracterización conceptual se apoya en las tres perspectivas teóricas revisadas, si bien pone énfasis en la dimensión crítica y sociocultural del constructo (Prior, 1998; Donahue, 2005; Gee, 2005; Ivanic, 2005).

En base a estas premisas que sustentan nuestra conceptualización, hemos elaborado una propuesta metodológica integradora que, por el momento, atiende solo a la manifestación de la voz en los textos. Se trata pues de un sistema de análisis de los textos escritos que debería ser útil tanto en el estudio de textos en construcción (borradores sucesivos) como en el de los textos finales. Nuestra pretensión es completar esta propuesta incluyendo en el análisis las representaciones cognitivas y afectivas de los escritores y lectores a lo largo del proceso de composición y de lectura. 
La propuesta actual contempla tres dimensiones de análisis estrechamente relacionadas pero independientes, cada una de ellas centrada en los aspectos que, de acuerdo a lo que acabamos de comentar, consideramos relevantes en la clarificación conceptual y definición de la noción de voz.

Así, la primera de estas dimensiones se refiere al análisis de los recursos discursivos que el escritor utiliza para hacerse más o menos visible en el texto. En lo que se refiere a esta dimensión, hemos optado por utilizar las categorías propuestas por Hyland (2005), considerando tanto su sencillez y amplitud como la validez de haber sido ampliamente utilizadas en diferentes disciplinas y con multitud de textos y géneros académicos diversos.

La segunda dimensión se refiere al análisis de la intertextualidad en los textos y las evidencias de negociación con otras voces. En este caso, las fuentes de las que partimos son mixtas; por un lado, nos apoyamos en algunas de las propuestas lingüísticas pero también en las que se desprenden de los trabajos sobre literacidad crítica y enfoques socioculturales.

La tercera de las dimensiones pretende analizar los aspectos estructurales los textos y para su análisis hemos recurrido a las propuestas de organización textual elaboradas por Swales $(1990,2004)$.

A continuación comentamos de forma más pormenorizada las características de cada una de estas dimensiones así como las categorías que incluyen.

c) Posicionamiento: Esta primera dimensión se refiere a la posición que adoptan los escritores en tanto que autores de su texto. Alude a los recursos discursivos que utiliza el escritor para posicionarse, de acuerdo a la definición de Hyland (2005). Tal como hemos avanzado, hemos partido de la clasificación propuesta por este autor para dar cuenta del posicionamiento que incluye cuatro categorías que resumimos a continuación.

i. 'Expresiones de elusión de compromiso o "hedges'2 (posible, tal vez, condicional)'. Señala el valor que debe otorgarse a una afirmación, considerando el grado de precisión o fiabilidad que le corresponde. Estas expresiones implican que una afirmación está basada en un razonamiento plausible más que en un conocimiento e indican el grado de confianza que se le puede atribuir (Hyland, 2005). Indican pues, la intención del escritor de aplazar su compromiso con respecto a una determinada información, permitiendo que sea presentada como una opinión en lugar de como un hecho acreditado o verdadero. Debido a que todos los enunciados son evaluados e interpretados a través del prisma de las suposiciones disciplinarias, los escritores deben calcular cómo presentar una afirmación, tanto para otorgarle más - menos fiabilidad como para protegerse en caso de su eventual refutación. Algunos ejemplos serían: "nuestros resultados sugieren..."; “[...] podría ser la causa de..." (Hyland, 2005: 52).

ii. 'Potenciadores-enfatizadores o boosters (claramente, obviamente, demuestra)'. Ayudan al autor a explicitar sus opciones y a expresar seguridad, a la vez que marcan el grado de implicación con el tema y solidaridad con la audiencia. Sirven para enfatizar información compartida y pertenencia a un grupo. El equilibrio entre hedges y boosters en un texto permite conocer cómo el autor se compromete con el contenido del texto y qué tipo de relación establece con sus lectores.

iii. 'Marcadores de actitud (de acuerdo con, preferente mente,desgraciadamente, afortunadamente)'. Indican la relación afectiva, más que epistémica, del escritor con las afirmaciones que hace en el texto.

iv. 'Auto-referencia'. Implica el grado de presencia explícita del autor en el texto, medida a través de la frecuencia en el uso de la primera persona y de los adjetivos posesivos. La ausencia o presencia de autoreferencias explícitas es generalmente una elección consciente en los escritores académicos expertos para adoptar una "identidad situada disciplinarmente" (Hyland, 2005: I8I).

v. 'Marcadores de implicación'. Son mecanismos que explícitamente se dirigen al lector, ya sea para focalizar su atención o para incluirlos en el discurso. En base a sus experiencias previas, los escritores pueden predecir las reacciones de los lectores y anticipar sus objeciones, sus dificultades de comprensión, entre otros. Se trata de guiarlos en su interpretación mediante preguntas y referencias al conocimiento compartido. Este proceso de evaluación de la audiencia ayuda a construir una línea efectiva de razonamiento y señala los modos en que el lenguaje está relacionado con contextos culturales y disciplinares específicos (Hyland, 2005). Entre estos mecanismos destacan el uso del plural que incluye a los lectores, los comentarios que interrumpen el discurso para asegurar el conocimiento compartido (paréntesis, notas al pie, etc.), las referencias al conocimiento compartido, los imperativos (considérese, note, imagine), las modalizaciones dirigidas a la audiencia (deberíamos, podríamos), los predicados que expresan los juicios del escritor (es importante entender...) y las preguntas. 
d) Intertextualidad: La segunda dimensión, a la que hemos denominado intertextualidad, alude a los recursos y mecanismos que utiliza el escritor para relacionarse con otros autores. Se refiere pues al diálogo que se establece con otros textos y autores que actúan como referentes explícitos. Nótese que el texto final solo puede dar cuenta de los referentes explícitos; evidentemente, una respuesta adecuada a una demanda de escritura abierta y compleja requiere no solo integración de lecturas (consideradas como textos-fuente explícitos) sino también integración de múltiples y variados conocimientos previos que contribuyen a construir la voz del autor. De todas formas, si bien entendemos que la intertextualidad no puede reducirse a los textos leídos o citados, en este caso hemos optado por unas categorías que solo dan cuenta de los referentes explícitos puesto que estos son los que un análisis exclusivamente textual, como el que estamos proponiendo, permite deducir.

Por otra parte, de acuerdo con Bazerman (2004), no se trata solo de mencionar los textos a los que el autor se refiere, sino también de poder analizar cómo los interpreta y los utiliza. Integrando estas premisas con las aportaciones de otros autores (Thompson \& Tribble, 200 I; White, 2004), optamos por analizar diferentes niveles de intertextualidad en función de la explicitud con la que el texto invoca otros textos que se concretan en las siguientes categorías que van de mayor a menor explicitud:

- Cita directa.

- Cita indirecta. Implica parafrasear y comentar una determinada cita.

- Mención de una persona, documento o enunciado. Exige explicar con las propias palabras lo que expresa una determinada fuente sin valorarla.

- Comentario evaluativo sobre un enunciado, texto u otras voces invocadas.

- Uso de frases o términos asociados a personas, grupos de personas o documentos.

- Uso del lenguaje característico de determinadas formas de comunicar, discutir con otros, o de tipologías de documentos: géneros, vocabulario, patrones de expresión.

e) Organización de la información: Esta dimensión se refiere al análisis del contenido desde la perspectiva de la organización o estructura de la información en los textos y, para su análisis nos hemos basado en la propuesta de movimientos - moves- elaborada por Swales (2004). A pesar de que la mayoría de autores que se han ocupado tanto de la precisión conceptual como de la aproximación metodológica a la noción de voz no han tenido en cuenta esta dimensión estructural de los textos, nos parece necesario incluirla en nuestra propuesta, puesto que contribuye a dejar clara la posición del autor a través de las opciones que este escoja para poner de manifiesto tanto la progresión temática como la conexión con sus propias ideas y las que provienen de la revisión bibliográfica. Precisamente ambos aspectos aparecen claramente en la propuesta de Swales $(1990,2004)$ como indicadores de las elecciones intencionales del escritor respecto a la forma de organizar el contenido y de mostrar las relaciones entre las ideas. Además, proponemos complementar este análisis con el detalle de todas las unidades temáticas del texto, para relacionar diferentes movimientos -organizativos- con los cambios temáticos.

\section{A modo de conclusión: Una propuesta de análisis de la voz en los textos académicos de estudiantes universitarios $y$ en investigadores}

A lo largo de este artículo, hemos revisado los aspectos conceptuales sobre los que se asienta el concepto de voz académica basándonos en el supuesto de que este concepto puede ser útil para ayudar a los estudiantes universitarios a escribir mejores textos $y$, en último término, para evitar el plagio cuando se enfrentan a la actividad de escribir en contextos académicos a partir de fuentes diversas. Nuestra pretensión no ha sido otra que la de proponer unas dimensiones de análisis que hemos comentado en el apartado anterior y que suponemos relevantes en la manifestación de la voz del autor en los textos, para su posterior análisis en estudios empíricos y además para su utilización en estudios de carácter educativo que tengan por objetivo la mejora de la escritura académica de los estudiantes tanto de secundaria como, preferentemente, universitarios.

En este sentido, el siguiente paso consistirá en la realización de un estudio empírico en el que nos ocuparemos de analizar cómo se evidencia la voz de diferentes tipologías de autores en el texto final producido. El corpus de análisis lo constituirán textos académicos elaborados por dos tipologías diferentes de autores: estudiantes de doctorado y doctores escritores expertos (miembros de la misma comunidad discursiva).

Suponemos que estos dos grupos exhibirán niveles de dominio diferentes en la gestión de su propia voz como escritores y nos interesa analizar 
Tabla I. Dimensiones, categorías y tipo de análisis en el estudio de la voz en los textos.

\begin{tabular}{|c|c|c|}
\hline Dimensión & Categorías & Tipo de análisis \\
\hline I. Posicionamiento & $\begin{array}{l}\text { I.I Expresiones de elusión de compromiso. } \\
\text { Hedges } \\
\text { I.2 Potenciadores-enfatizadores. Boosters } \\
\text { I.3 Marcadores de actitud } \\
\text { I.4 Autorreferrencia } \\
\text { I.5 Implicación }\end{array}$ & Discursivo \\
\hline 2. Intertextualidad & $\begin{array}{l}\text { 2.I Cita directa } \\
\text { 2.2 Cita indirecta } \\
\text { 2.3 Mención persona, documento o enunciado } \\
2.4 \text { Comentario evaluativo enunciado, texto u } \\
\text { otras voces invocadas } \\
\text { 2.5 Uso de frases o términos asociados a } \\
\text { personas, grupos o documentos } \\
\text { 2.6 Uso del lenguaje característico de grupos o } \\
\text { documentos }\end{array}$ & Discursivo y de contenido \\
\hline $\begin{array}{l}\text { 3. Organización de la } \\
\text { información }\end{array}$ & $\begin{array}{l}\text { 3.I Movimientos } \\
\text { 3.2 Unidades temáticas }\end{array}$ & $\begin{array}{l}\text { Estructural } \\
\text { de contenido }\end{array}$ \\
\hline
\end{tabular}

estas diferencias tanto en base al uso específico y preferente de unos recursos por encima de otros, como a la frecuencia y cantidad de este uso.

La Tabla I recoge las dimensiones que se analizarán, las categorías contempladas y los instrumentos utilizados en cada caso.

Esperamos que los resultados nos permitan establecer diferentes niveles de dominio en cada una de estas dimensiones para cada grupo de escritores.
Estos diferentes niveles de dominio pueden, a su vez, relacionarse con la construcción de la identidad de los estudiantes como escritores. Las implicaciones educativas que se derivan de estos resultados podrían facilitar el diseño de guías y actividades de escritura que incidiesen de forma positiva en la construcción explícita del concepto de autoría con la consiguiente desvalorización del plagio y la repetición en los textos académicos que producen los estudiantes. 


\section{REFERENCIAS BILIOGRÁFICAS}

Bakthin, M.M. (1986). Speech genres and other late essays. Austin, Tx: University of Texas Press.

Bazerman, C. (2004). Intertextuality: How texts rely on other text. En C. Bazerman \& P. Prior (Eds.), What writing does and how it does it. An introduction to analyzing texts and textual practices (pp. 83-96). Mahwah, New Jersey: Lawrence Erlbaum Associates.

Cadman, K. (1997). Thesis writing for international students:A question of identity? English for Specific Purposes, I6(I), I77-I88.

Calsamiglia, E. \& Tusón, A. (2007). Las cosas del decir. Manual de análisis del discurso. Barcelona: Ariel.

Castelló, M., Iñesta, A., Miras, M., Solé, I., Teberosky, A. \& Zannoto, M. (2007). Escribir y comunicarse en contextos científicos y académicos. Conocimientos y estrategias. Barcelona: Grao.

Corcelles, M. (2010). Construir la veu filosófica mitjançant l'escriptura col.laborativa: Una comunitat d'aprenentatge de la filosofia al batxillerat [en línea]. Disponible en: http://www.tesisenred. net/TESIS_URL/AVAILABLE/TDX-0923 I I 0-I24327/Tesi_Doctoral_Mariona_ Corcelles_I3_Set_20I0.pdf

Davies, B. \& Harré, R. (1990). Positioning: The discursive production of selves. Journal for the Theory of Social Behaviour, 20, 43-63.

Donahue, C. (2005). Student writing as negotiation. En T. Kostouli (Ed.), Writing in context(s) textual practices and learning processes in sociocultural settings (pp. 137-164). New York: Springer.

Fairclough, N. (1995). Critical discourse analysis:The critical study of language. Londres: Longman.

Farmer, F. (1995). Voice reprised:Three studies for a dialogic understanding. Rhetoric Review, I3(2), 304-320.

Gee, J. P. (2000). Identity as an analytic lens for research in education. Review of Research in Education, 25(I), 99-I25.

Gee, J. P. (2005). An introduction to discourse analysis. Theory and method. New York: Routledge.

Gee, J.P. (2006).A sociocultural perspective on early literacy development. En S. B. Neuman \& D. K. Dickinson (Eds.), Handbook of early literacy research (pp. 30-42). New York: Guilford Press.

Hunston, S. \& Thompson, G. (2000). Evaluation in text. Oxford: Oxford University Press.

Hyland, K. (1999). Academic attribution: Citation and the construction of disciplinary knowledge. Applied Linguistics, 20(3), 34 I-367.

Hyland, K. (200I). Bringing in the reader. Addressee features in academic articles. Written Communication, I8(4), 549-574.

Hyland, K. (2002). Authority and invisibility: Authorial identity in academic writing. Journal of Pragmatics, 34, I091-1 II 2.

Hyland, K. (2005). Stance and engagement: A model of interaction in academic discourse. Discourse Studies, 7(2), I73- 192.

Ivanic, R. ( 1998). Writing and identity: The discoursal construction of identity in academic writing. Amsterdam: John Benjamins. 
Ivanic, R. (2005). The discoursal construction of writer identity. En R. Beach, J. Green, M. Kamil \& T. Shanahan (Eds.), Multidisciplinary perspectives on literacy research (pp. 39l-4l6). Cresskill, NJ: Hampton Press.

Ivanic, R. \& Camps, D. (200I). I am how I sound: Voice as self-representation in L2 writing. Journal of Second Language Writing, I0, 3-33.

Kamberelis, G. \& Scott, K. D. (1982). Other people's voices: The coarticulation of texts and subjectivities. Linguistics and Education, 4, 359-403.

Lensmire, T. J. (1998). Rewriting student voice. Journal of Curriculum Studies, 30(3), 26I-29I.

Martin, J. R. (2000). Beyond exchange: Appraisal systems in English. En S. Hunston \& G. Thompson (Eds.), Evaluation in text (Pp. 142-175). Oxford: Oxford University Press.

Nelson, N. (200I). Writing to learn: One theory, two rationales. En P. Tynjala, L. Mason \& K. Lonka (Eds.), Writing as a learning tool: Integrating theory and practice (pp. 23-36). Boston/ Dordrecht: Kluwer.

Nelson, N. \& Castelló, M. (en prensa). Academic writing and authorial voice. En M. Castelló \& C. Donahue (Eds.), University writing: Selves and texts in academic societes. London: Emerald Group Publishing Limited.

Prior, P. (1998). A sociohistorical account of literate activity in the academy. New Jersey: Lawrence Erlbaum.

Prior, P. (200I).Voices in text, mind, and society. Sociohistoric accounts of discourse acquisition and use. Journal of Second Language Writing, I0, 55-8I.

Reuter, Y. \& Lahanier-Reuter, D. (2007). Analyzing writing in academic disciplines: A few concepts. LI-Educational Studies in Language and Literature, 8(2), 47-57.

Spivey, N. (1997). The constructivist metaphor: Reading, writing and the making of meaning. San Diego, CA:Academic Press.

Swales, J. (1990). Genre analysis: English in academic and research settings. Cambridge: Cambridge University Press.

Swales, J. (2004). Research genres. Exploration and applications. Cambridge: Cambridge University Press.

Thompson, J. P. \& Tribble, C. (200I). Looking at citations: Using corpora in English for Academic Purposes. Language, Learning \& Techonology, 5(3), 91-105.

White, H. D. (2004). Citation analysis and discourse analysis revisited. Applied Linguistics, 25( I), $89-116$. 


\section{NOTAS}

I El trabajo forma parte de un proyecto más amplio de mejora de la escritura académica y ha sido posible gracias a la financiación de sendas convocatorias de investigación de la FPCEE (Ref. FPCEEB-MQD/2008-0I0) y de la Dirección General de Investigación y Gestión del Plan Nacional de I+D+i del Ministerio de Ciencia e Innovación de España (Ref. SEJ2007-60868/EDUC).
2 No hay una traducción directa para el término hedges. Hemos adaptado la traducción de Calsamiglia y Tusón (2007: 170): "expresiones aproximativas de duda, rodeos o elusión del compromiso", reduciéndola a "expresiones de elusión del compromiso". 